\title{
ESTUDIOS SOBRE BASIDIOMYCETES. VII (HAYEDO DE MONTEJO DE LA SIERRA, MADRID)
}

\author{
G. MORENO, F. ESTEVE-RAVENTOS \& M. PEINADO
}

RESUMEN: Se estudian un total de 14 táxones que fructifican en el hayedo de Montejo de la Sierra (Madrid). Son nuevas citas para la micoflora española: Baeospora myriadophylla (Peck) Sing. y Eichleriella spinulosa Berk. E Curtis.

SUMMARY: Fourteen taxons that grow in the beech wood of Montejo de la Sierra (Madrid) are studied. Two of them are reported for the first time in Spain: Baeospora myriadophylla (Peck) Sing. and Eichleriella spinulosa Berk. E Curtis.

\section{INTRODUCCION}

Pretendemos en este trabajo, citar una serie de táxones recogidos en uno de los hayedos más meridionales de Europa, el encuadrado en Montejo de la Sierra (Madrid), para desde un punto de vista micológico ir anotando las especies micorrizógenas de Fagus sylvatica y precisando su cortejo florístico, tanto de hongos parásitos como saprófitos. Hemos recogido datos en el hayedo meridional de Pto. de la Quesera (Segovia) Checa \& Moreno (1982), y pretendemos compararlos cuando tengamos más información.

En los estudios hasta ahora realizados podemos indicar que el hayedo de Montejo de la Sierra, es una mezcla de melojar, Quercus pyrenaica, con haya y existe micológicamente mayor abundancia de las especies de melojar frente a las del hayedo, con un número muy pequeño de especies micorrizógenas de este. Los estudios se basan en las recolectas de los años 1981 y 1982 principalmente, años extremadamente secos en las estaciones favorables de aparición de fructificaciones de hongos, por lo que estos estudios se mantienen en la actualidad para completar lo más posible el número de especies del hayedo.

\section{MATERIAL Y METODO}

El material procede de diversos trabajos de campo efectuados por nosotros a la zona estudiada, tratándolo posteriormente con rojo congo amoniacal para recuperar su estructura, realizando después las fotografías en un microscopio Nikon modelo Optiphot con sistema 
incorporado de fotografía automática. Para la determinación de las especies nos basamos principalmente en la obra de Moser (1978).

El material está depositado en el Herbario particular de uno de los autores, H.GM, actualmente en el Departamento de Botánica de la Universidad de Alcalá de Henares, damos su numeración para cualquier consulta o posterior revisión.

\section{DESCRIPCION DE ESPECIES}

Baeospora myriadophylla (Peck) Sing.

Especie parecida en morfología a Baeospora myosura (Fr.) Sing. pero fructificando sobre madera de caducifolios, mientras que B. myosura es específica de estróbilos de coníferas. Se caracteriza microscópicamente (Lam. 1: 1, 2 y 3) por sus pequeñas esporas fuertemente amiloides y cilíndricas, midiendo de $3-4 \times 2-2,5 \mu \mathrm{m}$, presenta cistidios fusiformes a lageniformes.

Hábitat: rama de Fagus sylvatica, leg. G. Moreno (13-XII-1981), H. GM 2304 .

Bolbitius reticulatus (Pers. ex Fr.) Rick.

Especie de hábitat lignícola en el género, a diferencia de B. vitellinus que es un taxon coprófilo. Se caracteriza por su sombrero gris-violeta, con una amplia venación reticulada, que observado al microscopio (Lam. 1: 4, 5 y 7) tiene cutícula himeniforme con pelos que emergen y son pluricelulares y muy ramificados de forma variable.

Hábitat: tronco de Fagus sylvatica, leg. G. Moreno (15.V.1981), H. GM 2202 .

Collybia fuscopurpurea (Pers. ex Fr.) Kumm.

Se caracteriza por sus láminas marrón rosadas, con un pie marrón crema de base estrigosa. Las esporas son ovales a anchamente elípticas midiendo $6-7,5 \times 3-4 \mu \mathrm{m}$. (Lam. 1: 6 y 8).

Hábitat: humus hayedo, mezclado con melojo, leg. G. Moreno, H. GM 2484 .

\section{Crepidotus sphaerosporus (Pat.) Lange}

Presenta el sombrero blanquecino sin tintes ocráceos o amarillentos. Las esporas son ovales a esféricas, espinosas, midiendo 8-9 $\mathrm{x}$ 6-8 $\mu \mathrm{m}$. (Lam. 2: 15 y 16). Epicutis formada por hifas hialinas, cilindricas, ondulado-sinuosas; con fíbulas.

Hábitat: rama muerta de Rosaceae, leg. G. Moreno (11-XII-1981) H. GM 2275.

Crepidotus haustellaris (Fr. \& Fr.) Kumm.

Especie caracterizada por su pie lateral muy patente en estado adulto, a diferencia de otros Crepidotus. Al microscopio (Lam. 2: 9, $10,11,12,13$ y 14), presenta esporas ocráceas con poro germinativo manifiesto. Basidios bispóricos y pelos marginales cilíndricos-clavifor- 
mes, con fíbulas.

Hábitat: ramas de Fagus sylvatica descortezadas, leg. G. Moreno (6.XII.1981) H.GM 2251.

Eichleriella spinulosa Berk. \& Curtis

Se caracteriza por su fructificación resupinada de margen blanquecino y el resto de color carne-ocráceo, con abundantes espinas en el himenio de hasta $1,0 \mathrm{~mm}$ de longitud. Las esporas son alantoides de $15-18,5-(20) \times 6-8 \mu \mathrm{m}$. Presenta dicariofisos ramificados y tortuosos, siendo sus hifas fibuladas.

Hábitat: corteza tronco de Fagus sylvatica, leg. G. Moreno \& J.L. Mianjón (24.1II.1982), H.GM 2511).

Entoloma sinuatum (Bull. ex Fr.) Kumm.

Hâbitat: humus de hayedo y melojar, abundante en la zona, leg. G. Moreno (13.X.1979), H.GM 2425.

Exidiopsis grisea (Pers.) Bourd. \& Mre.

Fructificación resupinada gelatinosa, de color blanco-grisáceo en un principio. Las esporas son alantoides de 21-22-(25) x 6-8 $\mu \mathrm{m}$. Dicariofisos ramificados $y$ tortuosos, siendo sus hifas fibuladas (Lam. 2: 17,18 y 19).

Hábitat: corteza de Fagus sylvatica, leg. G. Moreno (8.X.1980) H.GM 2478.

Flammulaster carpophila (Fr.) Earle

Especie caracterizada macrocópicamente por su pequeño tamaño 0,5-1,2 cm de diámetro y con una cutícula farinosa-granulosa. Microscópicamente (Lam. 3: 20, 21, 22, 23 y 24) presenta los gránulos de la cutícula formados por células cilíndricas a ovales, a veces algo ramificadas. Las esporas son ovaladas con contorno amigdaliforme. Pelos marginales lageniformes, cubriendo la arista.

Hábitat: hojas de Quercus pyrenaica, lég. G. Moreno (13.X.1979), H. GM 2429.

Hebeloma radicosum (Bull. ex Fr.) Rick.

Especie muy característica en el género y fácilmente identificable microscópicamente (Lam. 3: 25 y 26), presenta esporas fuertemente ornamentadas, amigdaliformes y pelos marginales fuertemente cilíndricoclaviformes a cilíndrico-piriformes, con fíbulas.

Hábitat: humus bajo Fagus sylvatica, leg. M. García-Rollán (24.X.1976) H. GM 1648. 
Lyophyllum transforme (Britz.) Quél.

L. trigonosporum (Bres.) Kühn.

Especie bien caracterizada microscópicamente por sus esporas triangulares, únicas en el género (Lam. 3: 27, 28 y 29).

Hábitat: humus de Fagus sylvatica, leg.: G. Moreno (7.XI.1975), H. GM 772 .

Marasmius epiphyllus (Pers. ex Fr.) Fr.

Especie caracterizada por su hábitat; sobre peciolos y en los nervios de las hojas secas tanto de haya como de melojo. Es de pequeño tamaño, ya que el sombrero mide de $0,2-0,5 \mathrm{~cm}$, con el pie marrón y la parte superior blanquecina, y las láminas mal desarrolladas, tan sólo a modo de pliegues. Microscópicamente (Lam. 4: 30, 31 y 32) presenta esporas elípticas fusiformes y cistidios también fusiformes.

Hábitat: hojas de Quercus pyrenaica, leg. G. Moreno (13.X.1979), H. GM 2490 .

Omphalina rustica (Fr.) Quél.

Especie caracterizada por su pequeño tamaño, sombrero de 0,2$0,5 \mathrm{~cm}$, de color gris igual que el pie y las láminas, que se presentan bien desarrolladas. Microcópicamente (Lam. 4: 33 y 34) presenta esporas cilíndricas con vacuolas, midiendo 8-9,5 $54,5-5 \mu \mathrm{m}$.

Hábitat: taludes húmedos con algas verdes (hongo liquenizante), bordes de camino del hayedo, leg. G. Moreno (13.VII.1979), H. GM 2541

Psathyrella subatrata (Batsch. ex Fr.) Gill.

Especie bien caracterizada microscópicamente (Lam. 4: 35, 36, $37,38,39,40$ y 41) por la cutícula himeniforme con pelos delgados, largos y de gruesas paredes, de coloración marrón oscura. Las esporas son elípticas con poro germinativo inclinado a la cara externa. Cistidios únicamente marginales, lageniformes.

Hábitat: humus de Fagus sylvatica, leg. G. Moreno (13.VII.1979), H. GM 2587.

\section{DISCUSION}

De las especies estudiadas aparecen seis, de comportamiento lignícola saprófito, son: Baeospora myriadophylla, Bolbitius reticulatus Crepidotus haustellaris, Eichleriella spinulosa, Exidiopsis grisea, Hebeloma radicosum, esta última según la bibliografía consultada es la que parece más ligada a la vegetación de Fagus sylvatica, el resto son más amplias, generalmente de caducifolios. Cinco especies se muestran humicolas, son: Collybia fuscopurpurea, Flammulaster carpophila, Lyophyllum transforme, Marasmius epiphyllus Psathyrella subatrata, excepto esta última especie de corología y ecología no bien conocida en la Península Ibérica, el resto son táxones de amplia aparición en 
caducifolios, si bien Lyophyllum transforme tịene su óptimo en Coníferas. Crepidotus sphaerosporus apareció fructificando sobre ramas muertas de Rosaceae y Omphalina rustica se considera como un hongo liquenizante del grupo de los basidioliquenes.

\section{AGRADECIMIENTOS}

Nuestro más sincero agradecimiento al Profesor Lowy (Louisiana, U.S.A) por la confirmación de Eichleriella spinulosa y de Exidiopsis grisea, al Profesor Bon (Lille, Francia) por la confirmación de Bolbitius reticulatus y al Profesor Romagnesi (Paris, Francia) por la confirmación de Omphalina rustica.

\section{BI BL IOGRAF IA}

CHECA, J. \& MORENO, G. -1982- Contribución al estudio de los hongos que fructifican sobre Fagus sylvatica L. en el Puerto de la Quesera. Segovia. Bol. Soc. Micol. Cast., 7: 105-134.

MOSER, M. -1978- Die Röhrlinge und Blätterpilze (Polyporales, Boletales, Agaricales, Russulales). Gustav Fischer Verlag. Stuttgart, New York.

Trabajo presentado en la la Reunión Conjunta de Micología (1a Reunión del Grupo Especializado de Micologia de la SEM y V Jornadas de la AEEM), celebrada del 30 de septiembre al 2 de octubre de 1982 en la Universidad de Alcalá de Henares. Madrid. 


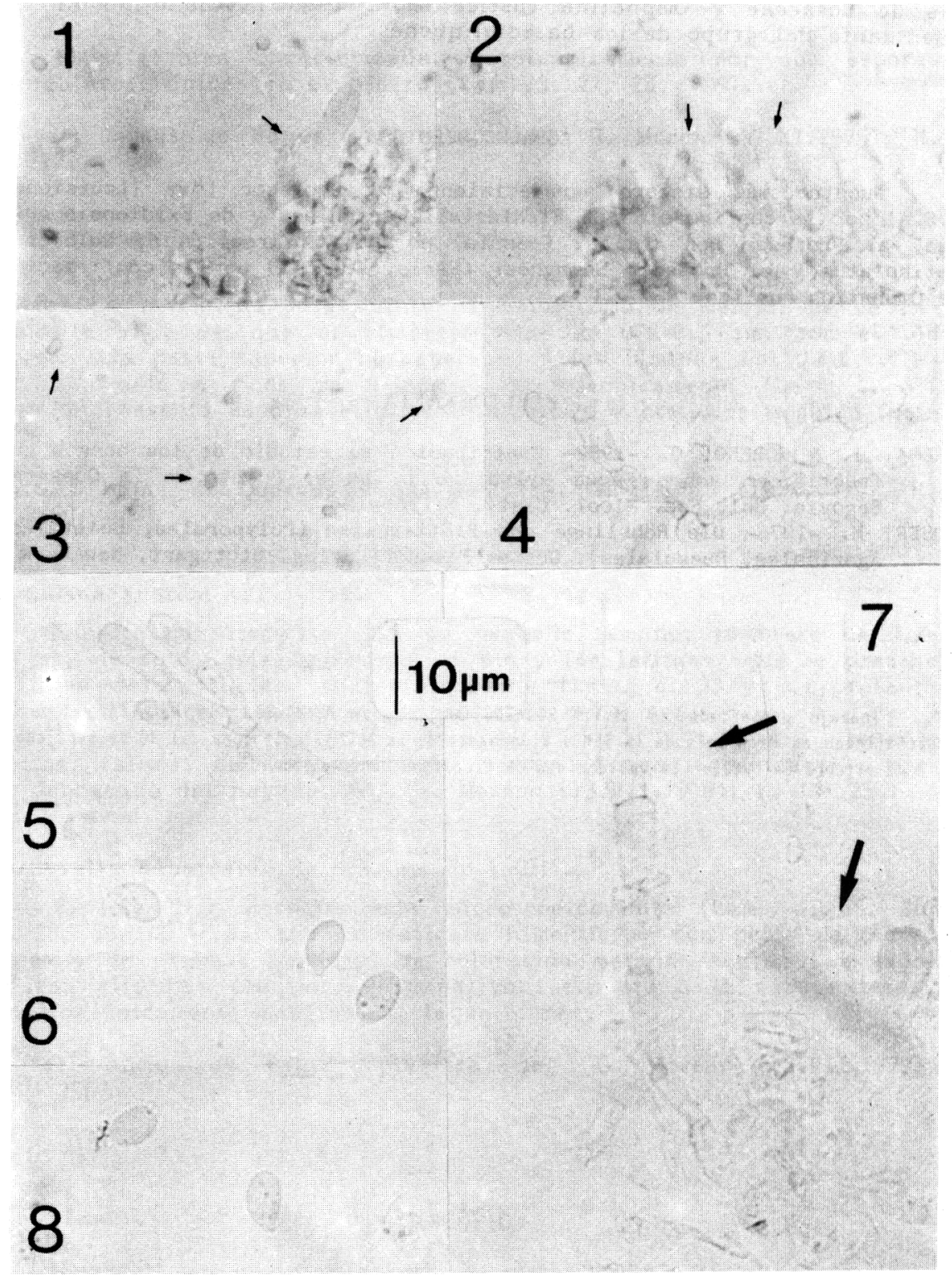

Lámina 1.- 1, 2 y 3: Cistidios y esporas de Baeospora myriadophylla (Peck) Sing.; 4,5 y 7: Cutícula de Bolbitius reticulatus (Pers. ex Fr.) Rick.; 6 y 8: Esporas de Collybia fuscopurpurea (Pers. ex Fr.) Kumm. 
Basidiomycetes de Montejo de la Sierr:

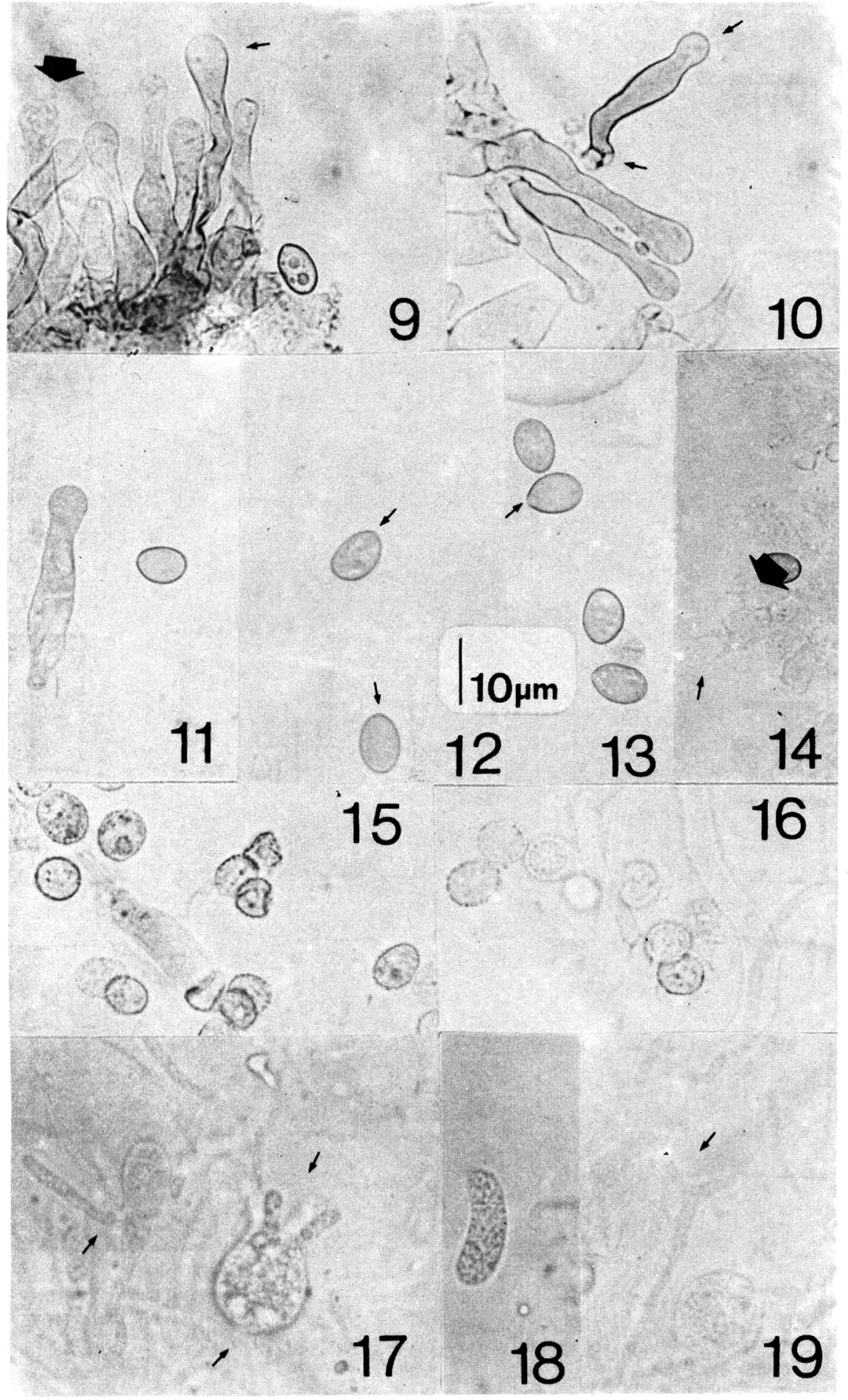

Lámina 2.- 9, 10, 11, 12, 13 y 14: Pelos marginales, esporas y basidio bispórico de Crepidotus haustellaris ( $\mathrm{Fr}$. ex $\mathrm{Fr}$.) Kumm.; 15 y 16: Esporas de Crepidotus sphaerosporus (Pat.) Lange; 17, 18 y 19: Basidio, espora y parafiso de Exidiopsis grisea (Pers.) Boud. \& Mre. 


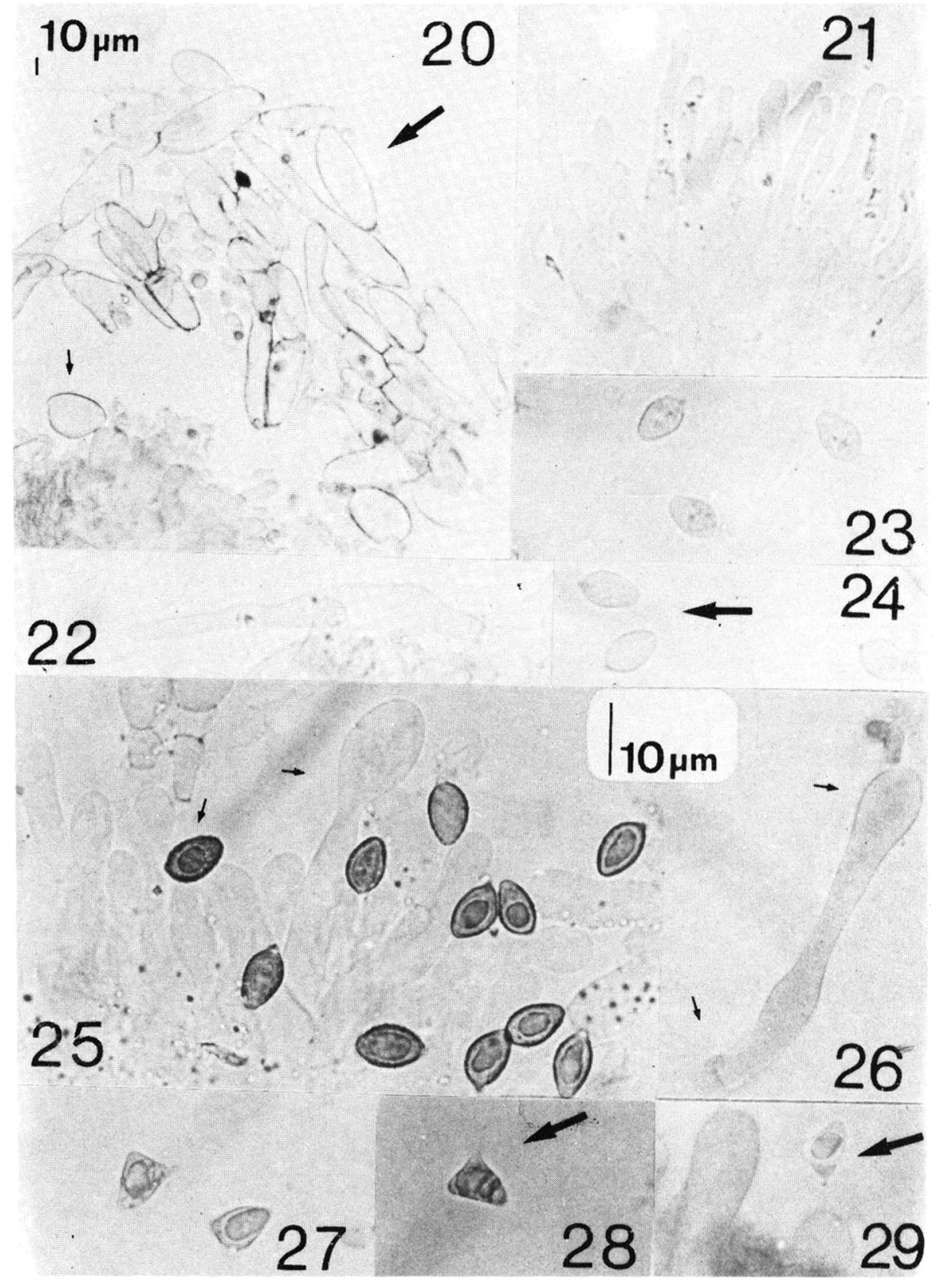

Lámina 3.- 20, 21, 22, 23 y 24: Cuticula, pelos marginales y esporas de Flammulaster carpophila (Fr.) Earle; 25 y 26: Esporas y pelos marginales de Hebeloma radicosum (Bull. ex $\mathrm{Fr}$.) Rick.; 27, 28 y 29: Esporas y basidio de Lyophyllum transforme (Britz.) Quél. 


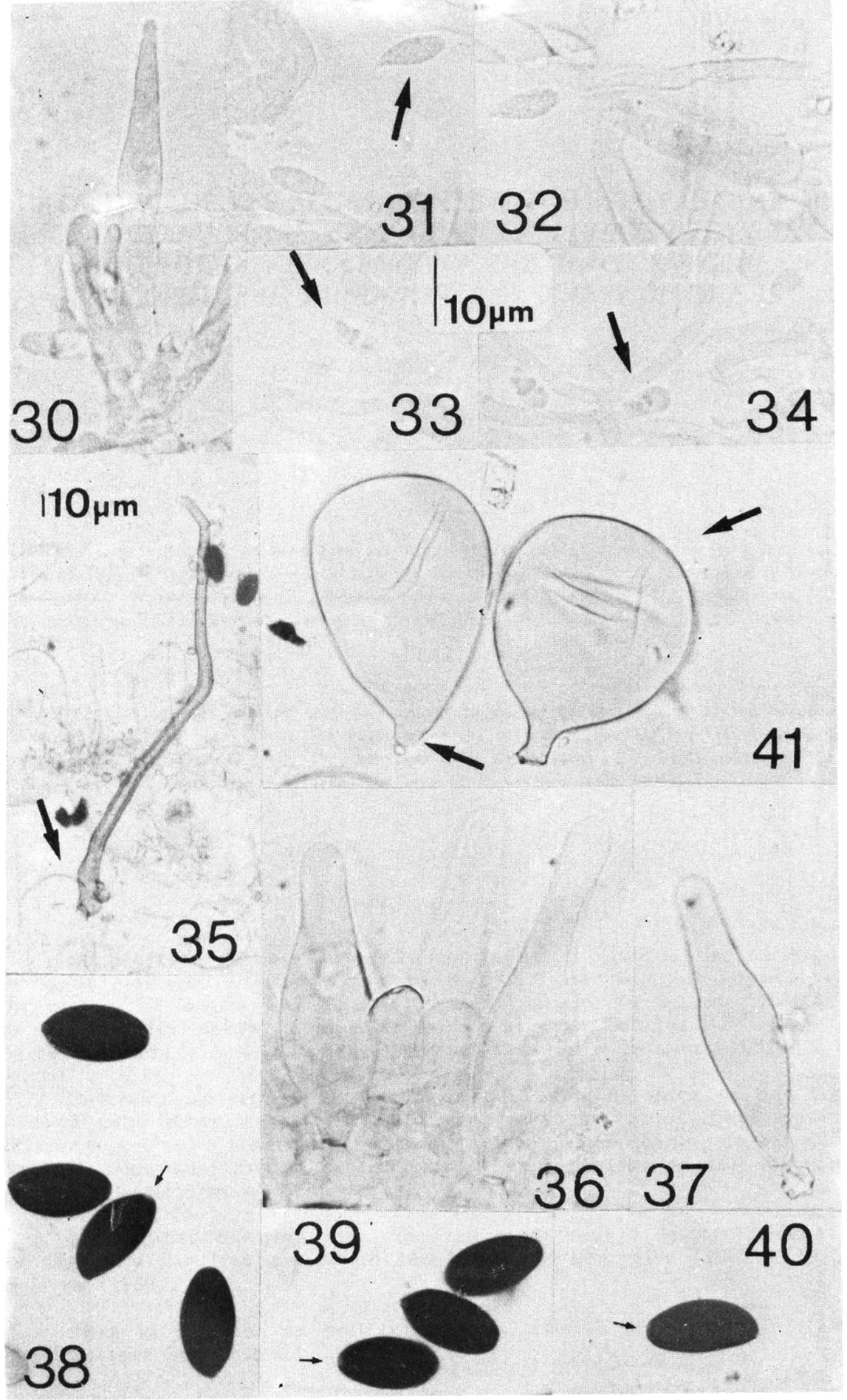

Lámina 4.- 30, 31 y 32: Cistidios y esporas de Marasmius epiphyllus (Pers. ex Fr.) Fr.; 33 y 34: Esporas de Omphalina rustica.(Fr.) Quél.; 35, 36, 37, 38, 39, 40 y 41: Cutícula, esporas, cistidios de Psathyrella subatrata (Batsch ex Fr.) Gill. 